definition of $f(x, y)$; representation of certain meromorphic functions as quotients.

The fourth lecture dealt with implicit functions under the following headings: Weierstrass's theorem of factorization; a tentative generalization of the foregoing theorem; algebroid configurations; single-valued functions on an algebroid configuration; connectivity and the Riemann manifold; parametric representation im Kleinen; solution of a system of analytic equations, Weierstrass's theorem; a general theorem; special cases of the foregoing; the inverse of an analytic transformation.

In the final lecture, Professor Osgood discussed the prime function, introduced by Klein in the theory of the algebraic functions and their integrals. That function is not a function on the given algebraic configuration, but depends on the homogeneous coordinates of an allied configuration. It is possible, however, to obtain a function on the given configuration which has a single zero and is completely analogous to the elliptic $\theta$ or $\sigma$ function.

Arnold Dresden.

\title{
THE VIENNA MEETING OF THE DEUTSCHE MATHEMATIKER-VEREINIGUNG.
}

THE annual meeting of the Deutsche MathematikerVereinigung was held in affiliation with the eighty-fifth convention of the association of German naturalists and physicians at Vienna, September 22-25, under the presidency of Professor K. Rohn.

The Germans fully recognize the importance of the social side of these annual meetings, and make ample provision for the pleasant entertainment of the guests. Although the program was a long one, frequent excursions were arranged and a reception or concert each evening.

The session of Thursday afternoon was devoted to the administrative affairs of the society. Reports of the status of the Encyclopedia, the Euler commission, and the International commission were read, and a briefer statement was submitted concerning the publication of various other works supported in whole or in part by the society. 
The sessions of Tuesday were held jointly with those of the sections of physics, geodesy, and astronomy at which the papers on applications of mathematics were presented.

The Vereinigung has now 770 members. The next annual meeting will be held in Hannover under the presidency of Professor C. Runge, of the University of Göttingen.

The following papers were read before the society.

(1) F. MEYeR (Königsberg): "Bericht über neuere, besonders durch Arbeiten von Gordan veranlasste Fortschritte der Invariantentheorie."

(2) E. MÜLleR (Vienna): “Eine Weiterbildung der Grassmannschen Ausdehnungslehre im Sinne der Invariantentheorie."

(3) G. KoHN (Vienna): "Zur Geometrie der Würfe."

(4) F. HoĉEvar (Graz): "Ueber den Zusammenhang zwischen den irreduziblen Teilern einer Form und einem linearen System von Nullstellen der Form."

(5) R. Weitzennвöck (Graz): "Invarianten bezüglich Untergruppen der allgemeinen projectiven Gruppe."

(6) E. BlaschKe (Vienna): "Aenderungen der Sterbewahrscheinlichkeiten mit der Zeit."

(7) L. G. Du Pasquier (Neuenburg): "Eine neue Anwendung der simultanen Differentialgleichungen in der mathematischen Theorie der Lebensversicherung."

(8) A. Einstein (Zürich): "Zum Gravitationsproblem."

(9) W. v. Dyck (Munich): "Ueber die Kepler-Manuskripte der Wiener Hofbibliothek."

(10) A. KoRN (Berlin): "Zur Frage der internationalen Vereinheitlichung wichtiger Begriffe und Bezeichnungen in der Potential- und Elastizitätstheorie."

(11) E. Waelsch (Brünn): "Zu den Minkowskischen Grundgleichungen der Elektrodynamik."

(12) R. Menмke (Stuttgart): "Ueber die zahlmässige und graphische Lösung eines Systemes von unendlich vielen Gleichungen in einer unendlichen Anzahl Unbekannten."

(13) C. Juel (Kopenhagen): "Ueber Elementarflächen."

(14) F. Engel (Giessen): "Lie's Invariantentheorie der Berührungstransformationen und ihre Verallgemeinerung."

(15) H. Liebmann (Munich): "Die Entwicklung der Lehre von den Berührungstransformationen."

(16) K. ZindLER (Innsbruck): "Ueber geschlossene Raumkurven." 
(17) H. Tietze (Vienna): "Ueber ein-eindeutige stetige Abbildungen von Flächen auf sich selbst."

(18) H. HAHN (Czernowitz): "Ueber stetige Abbildungen."

(19) E. HECKE (Göttingen): "Modulfunktionen von zwei Variabeln und ihre Anwendungen auf algebraische Körper."

(20) L. v. SchrutKa (Brünn): "Zur additiven Zahlentheorie."

(21) P. Koebe (Leipzig): "Wesen und Ziele der Kontinuitätsmethode."

(22) J. Plemeld (Czernowitz): “Ueber den Verzerrungssatz von P. Koebe."

(23) J. PlemelJ (Czernowitz): "Ueber die Abhängigkeit der Lösungen linearer Differentialgleichungen von den akzessorischen Parametern."

(24) F. Dingeldey (Darmstadt): "Ueber ein gewisses Integral und eine einfache Darstellung der Kugelfunktionen."

(25) J. RADON (Vienna): "Unabhängigkeit von Kurvenintegralen vom Wege beim Bestehen von Nebenbedingungen."

(26) W. Gross (Vienna): "Zur Theorie der unbestimmten Differentialgleichungen."

(27) R. Suppantschitsch (Vienna): "Ueber die Axiomatik der Methode der kleinsten Quadrate."

(28) E. HÄNTzscheL (Berlin): "Bedingungen für die Lösbarkeit eines Fermatschen Problems."

(29) O. Perron (Tübingen): "Ueber eine eigentümliche Schwierigkeit bei der Integration gewisser scheinbar sehr einfacher Differentialgleichungen."

(30) (Miss) E. Noether (Erlangen): "Ueber rationale Funktionenkörper."

(31) F. Nowther (Karlsruhe): “Zur Theorie der Turbulenz."

(32) R. KöNıg (Leipzig): "Arithmetisch-funktionentheoretische Parallelen."

Abstracts of all the papers except the first follow below; the numbers correspond with those in the list of titles above.

2. Professor Müller generalizes the concept of products of points, rays, and planes, as developed by Grassmann, to apply to a number of new combinations which he calls manifold products. Every combination of these products which represents a number is an invariant of the quantities appearing in the equations. They do not depend upon a definite 
normal form, a fact that presents many advantages. Finally, the relation between this method and other symbolic methods is pointed out.

3. Nineteen years ago Professor Kohn introduced the throw of v. Staudt as a fundamental concept underlying the projective properties of a geometric configuration. The most important advances made by the same author center about a principle of duality of this geometry, by means of which the throws of figures appear in pairs of reciprocal throws. The contrajective figures or figures of reciprocal throws form a complete analogy to the projective figures or figures of equal throws. In a series of examples the author shows that just those properties have been lacking in which figures of equal throws have been replaced by figures of reciprocal throws.

4. The known relation between the zeros and the linear factors of a binary form was extended by Professor Hocevar to apply to the totality of the points of intersection of a variety $F\left(x_{1}, \cdots, x_{n}\right)=0$ and a straight line in $R_{n-1}$. The process is illustrated geometrically, but the procedure is algebraic.

5. Professor Weitzenböck discussed the following topics: application of symbolic methods of the theory of projective invariants to the invariants of elementary geometry (motion and reflection); proof of the first and second fundamental theorems of the symbolic method, and of the finiteness of a complete system of invariants of motion; construction of such a system for the conic. The memoir is to appear in the Wiener Berichte.

6. The latest results of the statistics of insurance, that the probability of death does not agree with that expected from the theory based upon probabilities, makes a new establishment of the foundations of this science necessary. Professor Blaschke proposed a new derivation of the formulas and a new interpretation of the results. A fixed premium is the only equitable method of making payments.

7. In Dr. Du Pasquier's paper we start with a closed company $A^{\mathrm{I}}$ of insured persons, consisting of persons of the same age $x$, of the same sex, living contemporaneously in the same 
district, in the same occupation, and all married. Let the number of members be $y_{1}(x)$. In the course of time the number will grow smaller through three causes: death, death of the wife (or husband), invalidity. Those in the second category compose a new company $A^{\mathrm{II}}$, with conditions similar to $A^{\mathrm{I}}$, except that they are not married. Let this number be $y_{2}(x)$. This number may change by death, marriage, or invalidity. Those in the second set now belong to $A^{\mathrm{I}}$ again. A third company $A^{\mathrm{III}}$ of $y_{3}(x)$ persons consists of invalids (unmarried), and a fourth $A^{\mathrm{Iv}}$ of $y_{4}(x)$ married invalids. Relations exist between $A^{\mathrm{I}}$ and $A^{\mathrm{IV}} A^{\mathrm{Ir}}$, and $A^{\mathrm{IV}}$. These are expressed by means of differential equations of the first order, the constants determined by statistics.

8. In order to establish the theory of gravitation from the standpoint of the principle of relativity, Professor Einstein discussed two possible methods. The first is the principle of the constancy of the velocity of light; it appears as a covariant of linear orthogonal transformations. The simplest theory of this kind is that developed by Nordström: "Gravitations-Feld durch einen Skalar $\phi$ bestimmt."

In the second method, the velocity of light is assumed to be variable; the gravitation field is determined by a tensor. Instead of four-dimensional vector theory we now have the absolute differential calculus. The equations of the gravitation field are derived from the conservation theorems. They are covariant under any linear transformation.

9. The newly discovered Kepler manuscripts described by Professor v. Dyck concern the publication of the Tabulæ Rudolphinæ and of the Observationes Tychonii Brahe and essays on the problem of the reformation of the calendar. The manuscripts and discussion will soon be published in the Abhandlungen of the Bavarian academy of sciences. Professor v. Dyck also explained the new regulation concerning the examination of candidates for higher teachers of mathematics and physics introduced in the Bavarian school system.

10. In his report Professor Korn mentioned the efforts of the committee, composed of prominent mathematicians, astronomers, and physicists, to reduce the nomenclature and definitions of the theory of the potential, to a uniform basis. 
In answer to the letter of inquiry, sent to a large number of persons concerned: "What are the words and concepts for which a uniform definition is desirable?" a large number of replies have been received. From these data the committee has discussed the question of the greatest desiderata, and it is believed that by including the theory of elasticity and extending a carefully planned organization gradually uniformity can be accomplished. The committee is confident that in this way an immense service can be accomplished, not only toward understanding the literature, but also toward clearing up a number of grave misunderstandings which now seriously interrupt the further progress of the science.

11. Professor Waelsch's paper is a continuation of his article "Quaternions and binary forms in the MinkowskiGauss equations of thermodynamics" which has recently appeared in the Berichte of the Vienna academy; the present paper will soon appear in the same journal.

12. In the graphical solution of an infinite system of equations with an infinite number of unknowns, Professor Mehmke employed the methods of descriptive geometry, generalized for space of an infinite number of dimensions. The memoir will be published in the Zeitschrift für Mathematik und Physik.

13. Professor Juel defines an elementary surface by the property of being divisible into a finite number of parts of surfaces of the third order. It is easily proved that every continuous closed surface having a continuously turning tangent plane will be an elementary surface in case the locus of the point of contact of four-point contact lines divides the surface in a finite number of pieces. For example, every regular analytic surface is an elementary surface. In such cases the ordinary theorems concerning the singular points of plane sections and of contour curves are easily proved geometrically, without making any assumptions concerning derivatives of the second order. It is believed that this procedure will prove to be as fruitful in the study of surfaces as that of the oval and convex arcs has been in the plane.

14. The theory of contact transformations can, as was 
shown in the Wiener Berichte, volume 110, by S. Kantor, be more easily established by means of the bilinear invariant of the Pfaffian expression $\Sigma p_{i} d u_{i}$ and the Poisson symbol $(\varphi x)$, the latter to be interpreted as a covariant in plane coordinates. It was shown by Professor Engel that the entire theory of invariants of contact transformations can be expressed by means of any Pfaffian $\alpha_{1} d u_{1}+\cdots+\alpha_{2 n} d u_{2 n}$ that can be put into the normal form $p_{1} d x_{1}+\cdots+p_{2 n} d x_{2 n}$.

Moreover, the theory of groups of functions can be given a new interpretation by defining each group by means of the complete system the solution of which consists of the functions, and can all be included, as was also shown by Kantor, among the invariant properties of a linear partial differential equation of the first order in $u_{1 p}$ under contact transformation. This problem can also be reduced to Pfaffians.

15. Professor Liebmann reported on the development of contact transformations as follows.

The two sources for the study of contact transformations, systematically elaborated and brought into connection with the theory of continuous groups by Sophus Lie, are the canonical substitutions of mechanics and Plücker's line geometry. The most important lines of investigation at present are in the direction of more minute interpretation and completion on the basis of the geometry of "Speere" (Study), and new methods in the theory of invariants under the infinite group of all point and contact transformations. [See the Mathematical Encyclopedia, Article III D7 (German edition now in press).]

16. Consider a one-partite closed, finite space curve which has a single tangent and a single osculating plane at each of its points. Take in particular such a curve of index 4, that is, such that no plane can intersect it in more than four real points. Professor Zindler studies the curve by means of the wedge of a point $P$ on the curve, defined by passing half-planes through the tangent at $P$ and the other points of the curve. The applicability of the idea was shown in the case of curves without cusps, and a set of curves found such that the wedge for each point of the curve is acute. From this fact follows that the curve has four points of undulation and two principal secants, such that the osculating plane at 
either point of intersection of the curve and a principal secant must pass through the other. The paper will be published in the Monatshefte.

17. Professor Tietze proved that every continuous biuniform mapping of a simply connected surface on itself which preserves the indicatrix is a deformation, that is, that by means of just such representations the identity can be reached.

18. In his paper on continuous mapping, Professor Hahn proved the following theorem: In order that a plane point set $M$ be a continuous representation of a segment, it is necessary and sufficient that it be closed, limited, and connected, and that in each of its points $P$ the condition be fulfilled: to every positive $\epsilon$ belongs a positive $\eta$ such that if a point $A$ of $M$ lies within the region $\eta$ of $P$, then in $M$ exists a connected part, containing both $P$ and $A$ and lying within the region $\epsilon$ of $P$.

19. As Dr. Hecke was called away from Vienna during the meeting, his paper was not read.

20. Professor Schrutka's paper was an abstract of a more extensive memoir based on the dissertation of Professor Vahlen, which appeared in Crelle's Journal, volume 112, page 1. The purpose of the paper is to introduce simplifications and render more systematic the methods there developed. The entire memoir will soon be published as a monograph.

21. Professor Koebe explained the principle of the method of continuity by means of two examples of conformal depiction of simply and multiply connected regions, and pointed out the differences between the older method of limiting polygons and his own new method of open polygonal continua, founded on theorems of deformation. An extensive application of the principle is made in a memoir being published in the Mathematische Annalen: "Ueber die Uniformisierung der algebraischen Kurven IV und V."

22. Professor Plemelj showed that the coefficient $a$ in $f(z)=z+a z^{2}+\cdots$, in case $f(z)$ is simply represented in the 
unit circle, satisfies the inequality $|a| \leqq N<5$, wherein $N$ is probably equal to 2 . The inequality

$\frac{1}{2 N}\left[1-\left(\frac{1-|z|}{1+|z|}\right)^{N}\right] \leqq|f(z)|<\frac{1}{2 N}\left[\left(\frac{1+|z|}{1-|z|}\right)^{N}-1\right]$

can then be derived. In case $N=2$ we have

$$
\frac{|z|}{(1+|z|)^{2}} \leqq|f(z)| \leqq \frac{|z|}{(1-|z|)^{2}}
$$

as a simple expression of the theorem of deformability of Koebe.

23. In Professor Plemelj's second paper the solutions of the differential equation are expressed as integral functions of accessory parameters, of Laguerre genus zero when the parameters appear linearly. In this way the solutions of very diverse types of differential equations can be obtained, including boundary problems, theorems of oscillation, Klein theorems, etc.

24. Professor Dingeldey first determined the conditions under which the integral

$$
\int \frac{x^{m}}{\sqrt{a x^{2}+b x+c}} d x
$$

is algebraic, when $m$ is a positive integer and $a, b, c$ are real. If $a, b, c$ are all different from zero and $m$ even $(m=2 n)$, the equation of condition is of order $n$ in $b^{2}$ and $a c$; if $m=2 n+1, b$ is a factor, and the other factor is similar to the preceding. By putting $b^{2} \div a c=z^{2}$, the resulting equation in $z^{2}$ has only real roots, distinct and positive. It is closely related with the equations appearing in spherical harmonics, which leads to an easy and natural representation by means of a combinant. For example, we have

$$
P_{5}(z)=\frac{1}{5 !}\left|\begin{array}{lllll}
z & 1 & 0 & 0 & 0 \\
1 & 3 z & 2 & 0 & 0 \\
0 & 2 & 5 z & 3 & 0 \\
0 & 0 & 3 & 7 z & 4 \\
0 & 0 & 0 & 4 & 9 z
\end{array}\right|,
$$

and similar expressions for $P_{m}(z)$. 
An extensive abstract will be published in the Jahresbericht.

25. What conditions must a curvilinear integral

$$
\int\left(P d x+\sum_{k=1}^{n} Q_{k} d y_{k}\right)
$$

satisfy in order to be independent of the path of integration when the paths are restricted by certain differential equations

$$
\varphi_{a}\left(x, y_{1}, \cdots, y_{n}, y_{1}{ }^{\prime}, \cdots, y_{n}{ }^{\prime}\right)=0, \quad(a=1, \cdots, m<n) ?
$$

In order to answer this question Dr. Radon assumes that the differential equations do not possess an integral $\phi\left(x, y_{1}, \cdots, y_{n}\right)=c$. The important results are: If by means of the $\varphi_{a}=0$ no linear equation $a d x+\Sigma b_{k} d y_{k}=0$ can be derived, then $P d x+\Sigma Q_{k} d y_{k}$ must be a complete differential. If, on the other hand, such linear equations can be derived, then the expression $P d x+\Sigma Q_{k} d y_{k}$, after simplification by means of these equations, is reduced to a complete differential in the ordinary sense.

26. In a previous memoir, published in the Mathematische Annalen, Dr. Gross considered a system of differential equations which proved to be non-integrable, that is, unsolvable by means of the processes of differentiation and elimination, and the addition of arbitrary functions. In this memoir he shows how one can determine whether a given system belongs to the class of those previously discussed, and also how a solution (if there is any) can be obtained.

27. In establishing the method of least squares, in so far as the proof is not a variation of that given by Gauss, the attempt is made to select the most favorable linear combination from among the possible combinations of error equations. It is by no means evident why restriction to linear equations is made. Professor Suppantschitsch shows how this restriction is connected with simpler assumptions (axioms). The only quality of the functions employed that is required is continuity. The paper is being published in the Wiener Berichte.

28. In his correspondence with Jacques de Billy, Fermat 
takes up the question, handed down from Diophantos, to find an infinite number of pairs of rational numbers $(x, y)$ which satisfy the equation $y^{3}=a_{0} x^{3}+3 a_{1} x^{2}+3 a_{2} x+a_{3}$, the coefficients being rational. Professor Haentzschel discussed the derivation of the conditions under which the problem can be solved. The equation is satisfied if the equation $v^{2}=4 s^{3}+D$ has an infinite number of solutions, $D$ being the discriminant of the cubic form. This is the first condition. The second condition is that the equation $v=-c_{3,3}(x) / f(x)$ shall have a rational root (Haentzschel: "Ueber das Integral . . .," Berlin Mathematische Gesellschaft, 1910). This root is the first solution. The further investigation consists of three parts: (a) $D$ any rational number; (b) $D$ a rational square; $(c) D=0$. In each case there are two subdivisions, according as $a_{0}$ is or is not a cube.

29. Professor Perron showed that in order to obtain the complete integral of a differential equation of the form

$$
\left[\frac{d y}{d x}-f(x, y)\right]^{2}-[\varphi(x, y)]^{2}=0
$$

it is not sufficient to integrate the two equations

$$
\frac{d y}{d x}-f+\varphi=0, \quad \frac{d y}{d x}-f-\varphi=0,
$$

as has always been supposed. For example, the equation

$$
\left(\frac{d y}{d x}\right)^{2}-\left(x \sin \frac{1}{x}\right)^{2}=0
$$

has, besides the two integrals

$$
y_{1}=\int_{0}^{x} x \sin \frac{1}{x} d x, \quad y_{2}=-\int_{0}^{x} x \sin \frac{1}{x} d x,
$$

both of which vanish with $x$, an infinite number of others, including

$$
y_{3}=\int_{0}^{x}\left|x \sin \frac{1}{x}\right| d x .
$$

30. The treatment of fields of rational functions, as de- 
veloped by Dr. Emmy Noether, concerns fields whose elements are rational functions of $n$ variables. For these fields, the existence of a rational basis is proved, and the minimum basis defined. An application of the latter is made to the problem of constructing equations with a preassigned group. In conclusion, the existence of a basis of integrity is proved for a class of fields.

31. The hydrodynamic treatment of the phenomena of turbulence requires the determination of further stationary or of periodic solutions of the hydrodynamic boundary problem in addition to the known laminal flow. The treatment heretofore employed is confined to the immediate region of this flow and does not lead to the other real solutions. Dr. F. Noether showed that it is necessary to consider solutions having a finite variation from the laminal flow. The theory of the ramification of non-linear integral equations leads to the hypothesis that with a sufficiently large value of the Reynolds number such solutions ought to exist.

By means of Fourier series, the problem can be reduced to a boundary problem of ordinary non-linear differential equations. It is thus brought into close relation with a previous memoir of the author, discussed in the Berichte der Bayerischen Akademie, 1913, page 309.

32. Dr. König pointed out the analogies which exist between elementary arithmetic (theory of real numbers), advanced arithmetic (algebraic numbers) and the Riemannian theory of functions, and showed that the concepts of irrational numbers, ideals, and multiplicative functions are all extensions of a single idea. A more detailed abstract will appear in the next number of the Jahresbericht.

VIRGIL SNYdeR. 